\title{
Image processing as an integration tool between a dam safety system and smart grids
}

\author{
Alex Lopes de Oliveira ${ }^{\mathrm{a}}$, Luiz Carlos Magrini ${ }^{\mathrm{a}}$, Hae Yong Kim ${ }^{\mathrm{b}}$, Edvaldo Fábio \\ Carneiro $^{\mathrm{c}}$, Júlio César Pinfari ${ }^{\mathrm{c} *}$ \\ ${ }^{a}$ FDTE, R.Cap.Otávio Machado-525, SaoPaulo, 04718-000, Brazil \\ ${ }^{b}$ Escola Politécnica da USP, Av. Prof. Luciano Gualberto, tr. 3, 158, SaoPaulo, 05508-900, Brazil \\ ${ }^{c}$ CESP, Av. Nossa Senhora do Sabará, 5312, Sao Paulo, 04447-011, Brazil
}

\begin{abstract}
A widely used configuration to monitor minor displacements in concrete dams of hydroelectric power plants is based on the joint use of direct and inverted pendulums. By measuring the degree of inclination of the pendulum wire in both the $\mathrm{X}$ and $\mathrm{Y}$ axis, it is possible to measure the amount of displacement. In this work, a system capable of performing automated readings of the pendulum wires inclination, through the image processing technique, is presented. A processing methodology combining the calculation of the $\mathrm{x}$ component, the gradient signal and the Hough transform, was adopted for this purpose.
\end{abstract}

Keywords: Smart grid, dams monitoring, image processing, hough transform

\section{Introduction}

The Smart Grid technology has the potential to substantially improve the electricity service by increasing Reliability, Robustness and power grid Resilience, apart from contributing in the reduction of the environmental impact and cost decreasing. Reliability is achieved by making the system less prone to failures, while the robustness is achieved by increasing the MTTF (mean time to failure) of the power grid components. Resilience can be defined as the ability to withstand failures as well as quickly recover from abnormal conditions, in other words, the resilience impacts the grid reliability by making the system more reliable as it becomes more resilient. In countries whose energy matrix comes predominantly from hydroelectric generation, the concepts of reliability, robustness and resilience should also be applied mainly to the generation stage, where concrete and earth dams are also included. Continuous monitoring of structural efforts due to seasonal variations in the flow of the rivers that form the dam reservoir ensures uninterrupted operation of hydraulic generators installed at the dam due to the stochastic nature of hydro resources. With the objective to turn the power grid more resilient, CESP (Companhia Energetica de Sao Paulo) is developing a digital system called SiMoEs, for monitoring the structural safety of their dams; thus, contributing to the improvement of the power grid reliability, mainly during extreme weather conditions where their reservoir levels are below the minimum or during the existence of excess water being poured away. These unfavorable situations alter the distribution of the hydraulic forces within the dam's concrete blocks that have been in operation for many years.

Dams operating for many years are usually monitored manually through civil instruments, many of them embedded in the concrete blocks; thus, making their substitution expensive if not impossible. The low-cost solution currently developed by CESP considers the use of sensors and transducers to these civil instruments, which through computational intelligence simulate and take the measurements normally

\footnotetext{
* Manuscript received April 13, 2015; revised August 11, 2015.

Corresponding author. Tel.: +55-011-2528-3667; E-mail address: aoliveira@fdte.org.br.

doi: $10.12720 /$ sgce.4.3.209-215
} 
performed by a technician. To this end, a number of sensors, data acquisition modules, and a data communication network are being installed so as to enable and check if the hydro generation dispatch is consistent with the infrastructure capacity for a certain period of time; thus, avoiding any structural damage that may affect the reliability, robustness and resilience of the smart grid. To provide this functionality SiMoEs was designed as a versatile monitoring system that can be customized for any of the several company's dams, and implemented with a series of sensors and transducers that can be fixed to the civil instruments but in a way that does not interfere with the manual measurement, if required, in case of unsatisfactory measurements. The displacement, distances and level measurements are always converted to the same fixed coordinate system used at the manual readings, so as to enable comparison between them and also store them in a database to be incorporated into the time series.

The concrete blocks' vertical displacements usually are measured through civil instruments denominated direct and inverted pendulums that are simple, very reliable and accurate systems, aiming to monitor internal lateral deformations of concrete dams, dam foundations and abutments. They consist of stainless steel wire with a diameter of about $1 \mathrm{~mm}$, installed vertically between the dam crest and the foundation. The direct pendulum is fixed on the dam crest and kept tensioned by a weight that is kept immersed in a highly viscous oil tank in order to provide greater stability to the wire. The inverted pendulum is similar, differing only in the attachment point which is anchored in the rock, and is kept tensioned by a buoy floating in an oil tank at the dam crest. The pendulum manual reading is done by the wire position measurement at the horizontal plane according to the $\mathrm{X}$ and $\mathrm{Y}$ axes of a local frame of reference.

The readings provided by the sensors and transducers adapted to the incrusted concrete and earth instruments are periodically collected by IEDs (Intelligent Electronic Devices) and saved in a historical database after being transformed to engineering units, according to its constructive type. These outcome measurements must be quickly analyzed to evaluate the structural performance of the dam and its foundations, to detect any anomaly that could jeopardize operational safety. A parallel algorithm individually compare the processed measurements with theoretical predictions defined by the structural project, such as maximum or control values and also detects possible malfunctioning of any sensor or equipment that could affect the analysis and interpretation of the readings, and also may lead to investigations into the condition of concrete and earth dam structures.

\section{Direct and Inverted Pendulum}

The solution for collecting position and integration of measurements of direct and inverted pendulums was to determine the position of the steel wire through a graphic processing of images taken according to the $\mathrm{X}$ and $\mathrm{Y}$ axes of a horizontal plane. This is done so as to collaborate with the resilience of the smart grid, without physically interfering with the instrument, nor preventing the manual mode reading. To this aim, an IED equipped with developed software is in charge of capturing the two images according to the $\mathrm{X}$ and $\mathrm{Y}$ axes and process an image processing algorithm to identify the location of the pendulum's wire. These images are obtained from two low-cost high definition webcams; one of them points to the $\mathrm{X}$ axis and the other to the $\mathrm{Y}$ axis. Because the site does not have good lighting a LED array, triggered via software at the time of capturing both images, was installed.

The distance graphically obtained according to the $\mathrm{X}$ and $\mathrm{Y}$ axes is preprocessed by transferring this distance to an inertial frame of reference before being passed to the SCADA system. The SCADA system periodically collects the measurements of all sensors and transducers, and saves them into a corporate historical database. This IED is mounted in a metallic cabinet near the pendulum and communicates with the SCADA system through an Ethernet network via an optical fiber using the Modbus / TCP protocol.

\section{Methodology}

\subsection{Image acquisition}

The process of image acquisition is important for the methodology design process to ensure reliability 
and accuracy in the measurements, as the quality of the input data (captured images) presents a direct correlation with the metrological characteristics of the system. So, two main aspects should be observed during the image acquisition process: field of view and image resolution. These two aspects are important as they determine the accuracy of the system, which is determined by the physical size of the object corresponding to a pixel. A pixel is the smallest point that forms a digital image. The entire image is composed by thousands of pixels. The size of a pixel determines the smallest possible dimension to be computed by a measurement system based on image processing.

The field of view of a camera, or the extent of the area that can fit through the camera, is related to three factors: focal length, distance between the camera and the object, and the size of the photosensitive sensor. Unlike the distance between the camera and the object, which can be adjusted during camera installation, the other two camera parameters depend on the manufacturing features. The focal length is determined by the lens used in the camera, as the length is increased the narrower will be the field of view. The photosensitive sensor in a digital camera can be a CMOS (Complementary Metal-Oxide Semiconductor) type which has lower energy consumption, though it has low sharpness and sensitivity at low light. The CCD (Charge Coupled Device) type tends to be more sensitive at low light and create clearer images with good recreation of colors. But what really impacts the sharpness is not the kind of sensor, but the size of the sensor. The bigger the sensor, close to $36 \mathrm{~mm}$ x $24 \mathrm{~mm}$ (full-frame) which nearly matches the image seen by human eye, the more the light that is captured and the greater will be the clarity and field of view. However, cameras with large CCDs are significantly more expensive.

Image resolution can be characterized as the ability of an optical system to represent the details of a target from which images are being captured. Often, the resolution is measured by pixel counting, the convention is represented through the set of two whole positive numbers, wherein the first number is the amount of pixel columns (width) and the second is the amount of pixel rows (height).

Thus, the size of a pixel can be reduced in two ways: firstly, by increasing the image resolution of a given field of view, or secondly, by decreasing the field of view. In this project, the use of low-cost CCD type cameras was chosen, bearing in mind a future expansion of this system to all dam pendulums. Because of their low cost the CCD dimensions in these cameras are also small, resulting in a reduced sensitivity to the light, thus, having lower resolution. However, the poor light level reaching the CCD sensor can easily be compensated by using an auxiliary array of LEDs. To compensate the loss of resolution caused by using a low-cost camera, a camera model equipped with a macro lens type (150 mm) having a narrow field of view was chosen.

\subsection{Calibration}

To associate the pendulum actual positions to the $\mathrm{X}$ and $\mathrm{Y}$ distances captured by the cameras, it is necessary to execute a adjustment procedure that uses a calibration wire with a different color to that of the pendulum' steel wire. This calibration wire, in parallel with the original pendulum wire, is not permanently installed. The calibration wire should be mounted so that it can move freely in both X and Y directions. Its position is measured using the same measurement instrument used today to read the position of the real pendulum wire.

After completing the calibration, a calibration matrix is generated and the system is already capable of measuring the pendulum wire position. The calibration position of the wire viewed by a camera is calculated as the index of the column which intersects with the wire axis. The index is "center zero", that is, the center column receives the zero index. Columns to the right receive positive results whereas those to the left negative indexes.

\subsection{Detection of edges}

During the calibration and the measurement process it is necessary a correct identification of either the calibration or the pendulum wire edges. This detection process is critical due to the focus loss of the object which occurs in the border edge, as seen in Fig. 1. The Hough transform is a widely used method for edge detection, but it is recommended to use some preprocessing techniques to identify, even 
superficially, boundary contours to reduce the Hough transform computational time. In this system, a fast pre-processing algorithm based on a gradient pre-processing filter or derived image that is intended to detect sharper transitions between different regions (such as edges), was used.

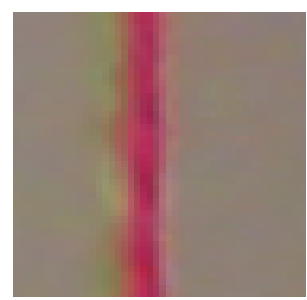

Fig. 1. Detail of wire edges.

\subsubsection{Preprocessing}

The calculation of the local gradient allows detecting boundaries in the image [1], as the edge is characterized by a high gradient region (Fig. 2).

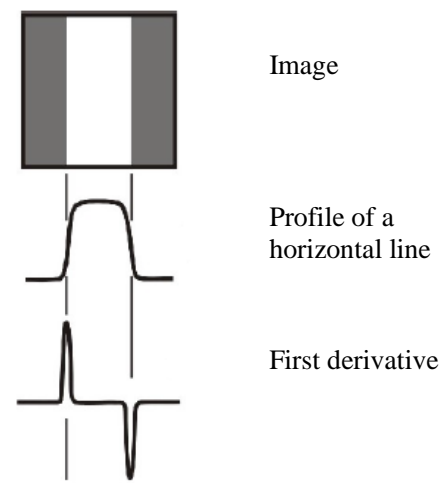

Fig. 2. Detection of edges by local gradient.

The gradient of a continuous function $\mathrm{f}: \mathrm{R}^{2} \rightarrow \mathrm{R}$ is defined in (1).

$$
\nabla f(x, y)=\left[\frac{\partial f(x, y)}{\partial x}, \frac{\partial f(x, y)}{\partial y}\right]
$$

Evidently, the above definition cannot be applied directly for a discrete image. In that case, its discrete version should be used. Typically, a G gradient for gray levels of a discrete image is used, as shown in (2).

$$
\nabla G(x, y)=[G(x+1, y)-G(x-1, y), G(x, y+1)-G(x, y-1)]
$$

After the preprocessing stage, an image highlighting the edges of wire will be obtained (Fig. 3).

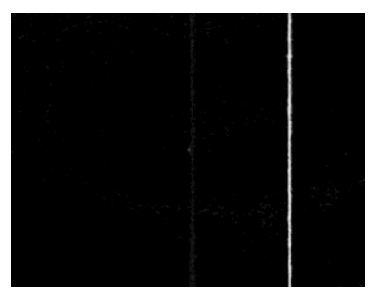

Fig. 3. Image highlighting the edges of the wire. 


\subsubsection{Hough transform}

The Hough transform, in its original form, is known since the 1960's [2], [3]. This technique has been applied for the solution of problems involving the detection and recognition of geometric shapes in digital images [4], [5]. Let $x$, y be the location in Cartesian coordinates of a black pixel in the image. The Hough transform converts the pairs of Cartesian coordinates $(x, y)$ curves in polar coordinates $(\rho, \theta)$ according to (3):

$$
\rho=x \cos (\theta)+y \operatorname{sen}(\theta)
$$

This transformation allows plotting a curve in the straight set passing through a pixel, then, for each pixel located at the blur of the steel wire edge there will be a different curve (Fig. 4).

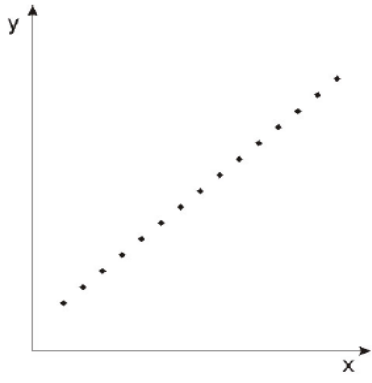

(a)

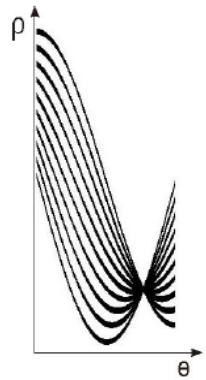

(b)

Fig. 4. a) x-y plane, b) parameter space [6].

where:

- $\rho$ represents the shortest distance of the line-passing through the pixel, to the origin (Cartesian coordinate);

- $\theta$ is the angle between the $\mathrm{x}$-axis (Cartesian coordinate) and the line between the coordinate origin and the nearest point of origin, passing through the pixel.

When two curves, represented in polar coordinates intersect at a point, for that specific coordinate ( $\rho$, $\theta$ ), there is a line passing through these two pixels. The Hough transform algorithm creates accumulation records which store the number of intersection in each polar coordinate $(\rho, \theta)$. The two coordinates that accumulate the greatest number of intersections are elected as the two lines that best represent the boundaries [7]. The system calculates the wire position calculating the average distance of the two edges of the wire pendulum (distance from the "central zero" set in the calibration process).

\section{Experimental Results}

The first field test with the automated reading system applied to pendulums was carried out on a direct pendulum, located in the concrete dam of the Sergio Motta hydroelectric plant (Fig. 5).

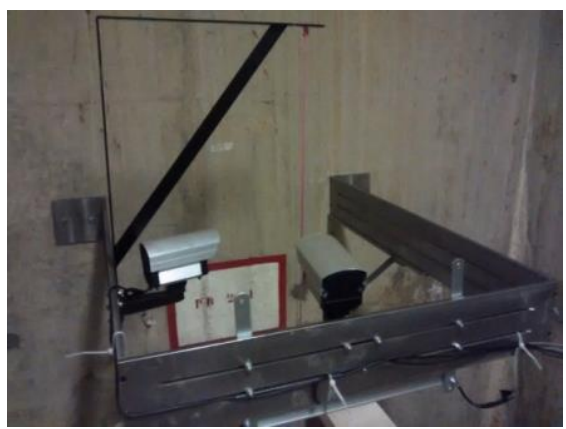

Fig. 5. Automated reading system installed in direct pendulum. 
The images of both $\mathrm{X}$ and $\mathrm{Y}$ axes were obtained from two cameras fixed on a metallic $\mathrm{U}$-shaped support, which in turn was fixed to the wall of the concrete dam (Fig. 6). The wires of both the calibration and direct pendulum pass through the metallic support; the latter only kept during the initial calibration.

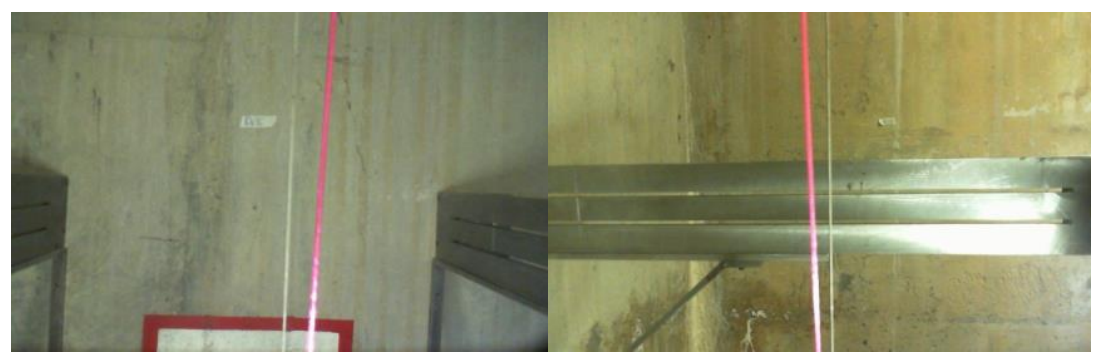

(a)

(b)

Fig. 6. (a) Image of $\mathrm{X}$ axis. (b) image of $\mathrm{Y}$ axis.

A number of tests to verify the system accuracy and its metrological repeatability (Table 1), as well as the execution time of the computational image processing (Table 2), were conducted.

Table 1. Average results obtained in 25 successive readings

\begin{tabular}{lcc}
\hline & $\mathrm{X}$ axis & $\mathrm{Y}$ axis \\
\hline Manual reading $(\mathrm{mm})$ & 3.00 & 3.50 \\
Mean reading $(\mathrm{mm})$ & 2.84 & 3.69 \\
Standard deviation $(\mathrm{mm})$ & 0.18 & 0.12 \\
\hline
\end{tabular}

Table 2. Execution time obtained in 25 successive readings

\begin{tabular}{lc}
\hline & Execution time of image processing \\
\hline Mean execution time $(s)$ & 3.80 \\
Standard deviation $(s)$ & 0.15 \\
\hline
\end{tabular}

The preliminary results showed to be satisfactory. As for the system accuracy and its repeatability, some additional evaluation tests and their application into an inverted pendulum still need to be done. From the preliminary results, it can be stated that a significant improvement in processing time was obtained, all this due to the pre-processing techniques previously presented, and also because larger images whose frame had dimensions equal to $640 \times 480$ pixels were used. The cost of the system, in relation to other automatic meter reading such as some automated coordinometers, is low; thus, becoming feasible for some other similar applications.

\section{Conclusion}

According to the study so far conducted, the measurement technique based on image processing using the Hough transform appears promising for the design of an automated reading system applied to direct and inverted pendulums. The measurements will be collected and saved in a database to thereafter analyze and evaluate the performance of the dam and its foundations; thus, enabling the detection of some anomalies that could jeopardize the operational safety. The study presents an important contribution for the safety of dams, where certain accidents may cause devastating consequences.

\section{Acknowledgements}

The authors gratefully acknowledge the financial support of CESP (Companhia Energetica de Sao Paulo) and ANEEL (Agencia Nacional de Energia Eletrica).

\section{References}

[1] Lee, JS. Refined filtering of image noise using local statistics. Computer Graphics and Image Processing, 1981; 15(4):380- 
389.

[2] Hough, PVC. Machine analysis of bubble chamber pictures. In: Proc. 16th International Conference on High Energy Accelerators and Instrumentation, 1959:5-10.

[3] Hough, PVC. Methods and means for recognizing complex patterns. U.S. Patent 3.069.654, 1962.

[4] Kimme C, Ballard D, Sklansky J. Finding circles by an array of accumulators. Commun., 1975; 18(2):120-122.

[5] McLaughlin RA. Randomized hough transform: improved ellipse detection with comparison. Pattern Recognition Letters, 1998; 19(3):299-305.

[6] Gonzalez RC, Woods RE. Digital image processing. 2nd ed. New York: Prentice Hall; 2002.

[7] Illingworth J, Kittler J. A survey of the hough transform. Computer Vision, Graphics, and Image Processing, 1988; 44(1):87116. 